\title{
A Thorough Insight to Techniques for Performance Evaluation in Biological Sensors
}

\author{
Subhas Meti ${ }^{1}$, V. G. Sangam ${ }^{2}$ \\ ${ }^{1}$ Dept of Instrumentation Technology, B V B College of Engeening \& Technology Hubli, India \\ ${ }^{2}$ Dept of Electronics and Instrumentation, Dayanand Sagar College of Engineering Bangaluru, India
}

\begin{tabular}{|c|c|}
\hline Article Info & ABSTRACT \\
\hline Article history: & \multirow{9}{*}{$\begin{array}{l}\text { The biological sensor has played a significant and contributory role in the } \\
\text { area of medical science and healthcare industry. Owing to critical healthcare } \\
\text { usage, it is essential that such type of sensors should be highly robust, } \\
\text { sustainable under the adverse condition and highly fault tolerant against any } \\
\text { forms of possible system failure in future. A massive amount of research } \\
\text { work has been done in the area of the sensor network. However, works done } \\
\text { in biological sensors are quite less in number. Hence, this manuscript } \\
\text { highlights all the significant research work towards the line of discussion for } \\
\text { evaluating the effective in the techniques for performance evaluation of } \\
\text { biological sensor. The study finally explores the problems and discusses it } \\
\text { under research gap. Finally, the manuscript gives highlights of the future } \\
\text { direction of the work to solve the research gap explored from the proposed } \\
\text { review of the existing system. }\end{array}$} \\
\hline Received Sep 15, 2015 & \\
\hline Revised Feb 9, 2016 & \\
\hline Accepted Feb 25, 2016 & \\
\hline Keyword: & \\
\hline Biological sensor & \\
\hline Fault tolerance & \\
\hline Performance evaluation & \\
\hline Validation & \\
\hline
\end{tabular}

Copyright (C) 2016 Institute of Advanced Engineering and Science. All rights reserved.

\section{Corresponding Author:}

Subhas Meti,

Dept of Instrumentation Technology,

B V B College of Engeening \& Technology Hubli, India.

Email: subhas.meti@gmail.com

\section{INTRODUCTION}

Biological and biochemical processes play a very important role in the field of medicine, biological sciences, and biotechnology. As it is very difficult to convert the biological data into electrical signals thus biosensors has been introduced for overcoming that difficulties. In the recent years various techniques and devices increased the usage of biosensors. In the year of 1962 Clark and Lyons designed the first bio-sensor [1] where they immobilized the glucose oxidase (GOD) on an aerometric oxygen electrode surface semipermeable dialysis membrane in order to examine a glucose concentrated sample. The process of making electrochemical sensors ( $\mathrm{pH}$, polarographic, potentiometric or conductometric) have been discussed by these two authors where they have added that "Enzyme transducers as membrane enclosed sandwiches". According to the definition given by IUPAC "A bio sensor is a self-contained integrated device which is capable of providing quantities and analytical information". The Fig 1 in background section discusses about various components of a bio sensing device which is mainly a collection of (a) a bio catalyst which converts the substrate or analyte to a product. (b) The transducer used to determine the chemical and biological reaction between ligand and analyte and converts it into an electrical signal. The final output is passed through an (c) amplifier (d) processor and finally (e) displayed on the screen [2]. It is found that at present the concept of bio sensors obtained huge attention of many researchers in different areas of application which include the bio medical industries, bio technology, pharmaceutical and environmental applications and the diagnostic for health related purposes. The functionality of a biosensor includes that it is a device which is used for observing, checking and keeping a continuous track of bio molecular interactions in real time scenario. In a bio sensor structure one of the components which is known as 'ligand' or the 'receptor' immobilized on the sensor chip, on the other hand the component of the solution which binds with the 
immobilized ligand or receptor is usually called as the analyte. The analyte presents in the solution phase. As the functionality of the analyte defines that it binds to the immobilized ligand and Bio-receptor. Bio-receptor and transducer are the most important components of biosensor (Figure 1). Researchg methodology is discussed in Section-2. The Researchh and discussions is discussed in Section-3. And Section-4 concludes the paper with brief discussion of future direction of research.

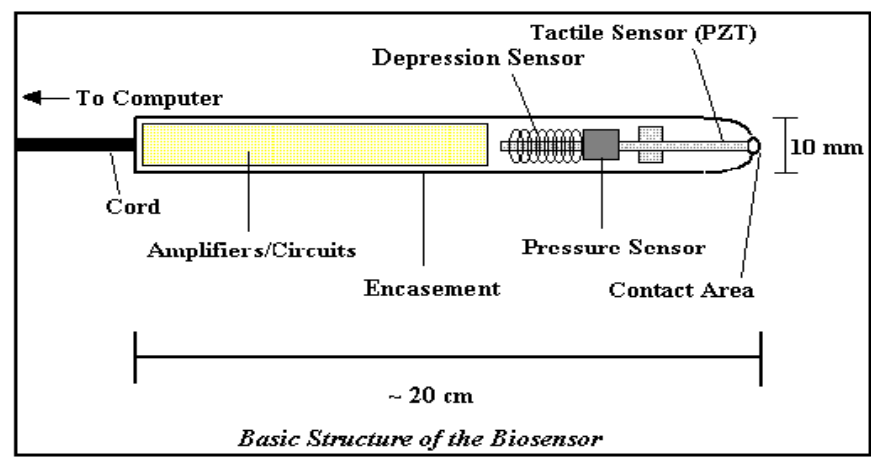

Figure 1. Components of Biological Sensor

\subsection{Background} as follows.

There are two different types of steps associated with the bio-sensor activities which are discussed

\subsection{Recognition Step}

In the recognition step, the immobilized biological element that is referred as 'Ligand' can recognize or detect the Analyte, which can be present either in the solution or the atmosphere. Immobilized elements can be proteins, antibodies, receptors, and enzymes, etc. The analytes which bind to these ligands can be antigens, drag molecules, protein substrates, etc.

\subsection{Transducing Step}

Analyte-receptor binding on the biosensor chip surface generates a signal that can be measured and analyzed. The receptor is designed where it has a close contact with the transducing element. This transducing element converts the analyte-receptor binding event into a quantitative optical or electrical signal. The generated signal can be either a. a change in the resonance unit, b. A change in the UV or IR absorption c. Change in Mass d. Change in electrical properties.

The intensity that is present in the biosensor generated signal found to be inversely proportional to the concentration of the analyte. The main components for designing and developing biosensors are an electrochemical transducer. Low costs, simple design or small dimensions can be achieved by electrochemical transducers. The design concept of the biosensors also based on gravimetric, calorimetric or optical detection techniques. It is found that the biosensors are classified according to the transducing elements as well as electrochemical, optical, piezoelectric and thermal sensors. Electrochemical biosensors also can be categorized potentiometric, amperometric and conductometric sensors. There are various application areas of biosensors that are a clinic, diagnostic, medical applications, process control, bio reactors, quality controls, defense research, and development, etc. A few advantages of biosensors are listed below:

- They can be applicable for measurement of nonpolar molecules that do not reply to most measurement devices.

- Biosensors are specific as a various immobilized system are used for designing them.

- Quick and enormous control is possible with biosensors.

- Response time is short (typically less than a minute) as well as

- Practical implementation can be done.

Various areas of applications that include the bio-logical sensor deployments are medical applications, rapid and accurate determination of chemical and biological agents that could be useful for national security, etc. In both chemical and national securities, various applications are used for detecting viruses and pathogens in dilute concentrations as the pathogens could be more life-threatening in contrast to security applications rather than medical applications. The identification of exotic and lethal diseases mostly associated with the biological weapon programs [3]. 


\subsection{The Problem}

This section discusses various issues related to wireless communication of biological sensors which are uniquely and fundamentally dissimilar from other sensor networks applications. There are various challenges arises in the field of biological sensor network which makes wireless networking significantly different from other networks. The following are the research issues of Biosensor Networks.

- Low Power: As it has been observed in the study of [4] that wireless sensor network whether it is biomedical or otherwise have energy consumption issues. As the biomedical sensors are also battery driven devices, thus they also have limited power source. This issue requires serious attention for enhancing supply in wireless biological nodes.

- Limited Computation: Computation capacity in bio sensors has become very limited and less effective due to very less amount of power supply. It is said by [5] the amount of computation which is possible with the use of biosensors is significantly less than traditional sensors.

- Material Constraints: As biomedical sensors are implanted in the human body so the size, shape and properties of a material should be examined properly [6].

- Continuous Operation: As biosensors are designed to have the potential to operate on limited battery power, so many researchers put their full effort for extending the battery life of a biosensor for their continuous operation on the human body. As it is a very low power device so that the frequent placement and adjustment of this device can cause some risk and functional disorders in human organs [7].

- Robustness and Fault Tolerance: The study of [8] says that the biological sensors are expected to be robust in nature as it is not possible to surgically adjust a biosensor every week. On the other hand fault, tolerance capability should be present in every node of the biosensor network thus malfunctioning of one individual node should not disturb the whole system or network.

- Scalability: It is not clear that a sensor or bio sensor network will be able to handle a growing amount of work in a distributed manner or not as it is also not found after so many investigations that how many sensors are needed to be placed to make a whole system functional [9].

- Security and Interference: Security and interference issues are considered as most important issues of the sensor network as sensor network is infrastructure less and use Ad-hoc mechanism for communication so that currently many researchers are investigating which topology might be suitable and most effective for Biosensor Networks [10].

- Regulatory Requirements: Design and safety must be fundamental features of biosensor network development. As bio sensor prototype devices are implanted in an organ cell of the human body so that wireless transmission of data should not harm the surrounding tissues [11].

The study of [12] highlights various noise processes and limits on the performance of biosensor networks. A comprehensive stochastic model has been proposed which describes the measurement uncertainty, output signal, and limitations associated with detection technique that is based on empathy based biosensors. It is also found that the biochemical events within the bio sensor platform have been designed with a Markov stochastic process to optimize the noisy signal transductions. This approach has been used for evaluating the output signal and the SNR (Signal to Noise ratio), noise figure, and detection of dynamic range for affinity-based biosensors have been created methodically.

- Generation of target specific signal: To produce the target specific signal, the target systematic computational analysis of data or statistics affects the recognition layer by interacting with through investigation into the crime and also participates in transduction process. Various probabilistic mass transfer processes have been raised to dominate the analyte motion. It has been observed that the collisions between analyte molecules and probes are very much probabilistic and deals with numerous uncertainties to the bio sensing procedures [13].

- Transducer and readout Circuitry: After analyzing various probabilistic mass transfer processes, it is found that the transducer and readout circuitry add more noise in the Probabilistic and stochastic processes [14].

- Concentration of the nonspecific Analyte: If the concentration of the non-specific analyte becomes much higher than the target analyte, non-specific bindings may dominate the measured signal and cause some effects which limit the minimum detectable limits (MDL) of the biosensor platform [15].

- Analyte Motion: Molecules cells many other things that are considered as analytes can be dipped and submerged in the aqueous mediums of bio sensory platforms cause thermal fluctuations. In general sensory platforms are considered as electromagnetic or mechanical forces. It is observed under the microscope that thermal fluctuation of a particle leads to follow the characteristics of typical random walk processes, as an example, it can be said that Brownian motion is the effect of subsequent diffusive spreading phenomenon or occurrence in a microscopic system. Statistical analysis associated with the

IJECE Vol. 6, No. 3, June 2016 : 986 - 994 
motion of individual analyte molecules subjected to many challenging situations apart from using the continuity equation formulation [16].

\subsection{The Proposed Solution}

This section talks about the data processing of biological sensors in a descriptive manner. Bio sensor data can be affected by the position on the resonance unit scale, noise, nonspecific responses and other objects that complicate the further processing of the data. The study of [17] highlighted that raw data need to be processed to ensure the capability of being compared. It has been also found that many SPR - based data processing techniques have been introduced. The study of [18] discussed that the most common commercially available SPR based bio sensors are bia-core 2000 and 3000 systems. The SPR based bio sensors are fortified with a four flow-cell fluidic system where one flow cell is used as a reference to subtract possible nonspecific signal. It is also found that several software packages are available for simplifying the datasets for biacore experiments e.g. Scrubber etc. Raw data which are obtained by the Biacore experiments are spread for different units of flow cell this process follows the mechanism of RU (Response Units). The Data-processing of Biological sensors can be classified into two methods.

\subsubsection{Data Aggregation}

In the recent times, the usages of bio sensors combine processing of different kind of data. Data aggregation is a process of extracting valuable information or accumulating information from the raw databases that are obtained by the biosensors. Data aggregation process of Biological sensor networks where microorganism identification has been done with the technique of spot color analysis. The experimental raw datasets have been evaluated with the decision processor to prepare a combined microorganism identification datasets [19].

\subsubsection{Existing Studies of Data Aggregation in Bio Sensor}

This sub section highlights various existing studies towards Data Aggregation of biosensors till date. The study of Halir et al. [20] presented a coherent detection scheme based on integrated optics that could be used to enable the unambiguous readout of the optical phase with a constant sensitivity. Experimental outcomes show the effectiveness of the proposed system and Phase shifts are considered as a performance parameters. A systematic analytic and numerical study has been proposed by $\mathrm{Wu}$ et al. [21] which includes the detection limit of a refractive index sensor. The proposed study applied coupled mode theory and investigated implementation results where wavelength $(\mathrm{nm})$, Time $(\mathrm{T})$ have been used as performance parameters. Horeman et al. [22] developed two dynamic bimanual positioning tasks that require adequate motion control as well as force control. The aim of the proposed study is to investigate the added values of force parameters with respect to time parameters where 10 of the 13 parameters showed a significant difference between groups. It is found in the study of Mehta and Zaghloul [23] that a tuning of the optical nano antenna in the visible spectrum using graphene has been introduced where a dipole structure for the nano antenna is considered with the reasoning wavelength. The FDTD simulation results have been verified with the experimental outcomes. Impedance and wavelength have been taken as performance parameters.

\subsubsection{Data Fusion}

Data fusion represented the process of enhancing multiple data and knowledge about a real object and mapped them into a consistent, accurate and useful representation. The Final processed data is sent to the base station.

Existing Studies of Data Fusion in Biosensor: This section gives a better overview about the existing studies associated with the Data Fusion of Biosensors. It has been found in the study of Blasch et al. [24] that extraction of features from information has been performed with the use of pulse coupled neural network. The proposed method extracts a feature from images. Different types of mathematical models have been explained to achieve an efficient feature based sensor fusion. Simulation results show target in an image and the effectiveness of Pulse Couple Neural Network (PCNN Model). Rahman and Marin [25] presented a particle swarm optimization (PDO) based algorithm for finding the optimal sink position. Relay nodes are introduced for optimizing the geometric network deficiencies. The experimental outcomes show the Lifetime comparison of relay nodes on size of the area and optimal sink location. The improvement of data utility by the optimal scheduling algorithm has been proposed by $\mathrm{Hu}$ et al. [26] where Simulation results show the performance analysis of virtual sensor scheduling algorithms. Performance analysis also shows the network utilities versus the number of time slots in a large scale network. 


\section{RESEARCH METHOD}

In general, the biosensor should be viewed as a biophysical instrument and, as with any highresolution technique, the better the reagents, the better and the results. At present, the performance validations of the biological sensors are found to be as following:

\subsection{Datamining}

Datamining is the process of extracting a typical and unique set of information called as knowledge from the given set of data. It consists of various conventional algorithms which is responsible for doing so. Usually, the amounts of the information extracted from the biological sensor are quite massive and it is extremely important to retain the reliability when processing the data. The study conducted by the author in [27] emphasized about datamining approach on bioinformatics as microbiology. The study conducted by the author in [28] has also signified the importance of datamining techniques. The prime reason behind it is that a set of statistical operations can be performed on the data generated from the biological sensors and a higher degree of knowledge could be extracted from such data formation.

\subsection{Machine Learning}

As discussed earlier that machine learning approaches can tackle the higher degree of complexities in the data being generated from the body sensor. The study conducted by the author in [29] has also discussed the significance of machine learning techniques.

It is found that adaptive thresholds are used for detecting failures and eliminate noise from the biosensor transducer generated signals. A failure event is usually detected by comparing the predicted and measured performance of the sensor. Where the comparison of the measured and predicted performance leads to the building of a residual. The residual evaluation is done by thresholds and then identifies the symptoms that are then analyzed for finding the associated fault.

Ideally, the residual is zero when there are no faults and not zero when a fault is present. It is also found that sometimes the residuals result greater than zero even when no fault is present. This may be due to

- Noisy measurements

- Unknown Disturbances

- Uncertainties in the models.

Fault detection mechanism is used to detect faults present in the biosensors. It is very essential to identify the faults in the biosensors as it is implanted in the human body and interacts with various chemical and biological reactions for producing reliable and crucial information about various parameters present in the human body. Section VI discusses the various validation techniques used to detect the fault in biosensor networks [30].

\subsection{Fuzzy logic based Techniques}

It can be seen that quantitative analysis of dopamine in the samples of urine and plasma is very essential as it is very useful for curing various health diseases like ganglioneuroma, schizophrenia, manicdepressive psychosis, burn out symptoms etc.As the presence of dopamine in a solution requires an enzyme reaction thus the experiment induced temperature also affects the output signal of biosensor. The paper of Ranglova et al. [31] presents a fuzzy based approach of biosensor input/output necessity Simulations performed on various software's confirms the high accuracy of fuzzy logic method. Relative errors which have been come out with the calculation of experimental data, used for the legitimacy of the proposed technique. It is found in the study of Singh et al [32] presents an optimized Self organized Fuzzy Logic controller for $\mathrm{pH}$ control with the use of performance correction table. It the controller performance is poor then this proposed mechanism involves a penalty for the output membership functions. A generalized fuzzy logic based approach for energy aware routing in sensor network has been developed by Haider et al [33] in order to optimize the energy consumption issues of sensor networks. This approach is soft and tunable and it can adjust with different types of sensor nodes having different energy issues.

\subsection{Game theory based Techniques}

Various game theory based validation techniques also found after analyzing so many research papers, this section highlights some of the significant game theory-based approaches for sensor networks. Koltsidas et al [34] designed a game theoretical modelling of clustering for sensor networks. The analysis has been performed in the basis of non-cooperative game approach where sensor behaves selfishly for preserving its energy. The proposed Clustered Routing for Selfish Sensors -CROSS) has been compared with various popular clustering techniques thus the simulation result shows the efficiency of the techniques with respect to performance parameters such as Number of Players (Nodes), Probability, Network Lifetime, Parameter ? , No of nodes alive and number of nodes around. The proposed study of Agah et al [35] proposed a protocol 
which is based on game theoretical approach. The main objective of the proposed study to design a protocol which recognize the presence of nodes that agree and fails to forward the data packets over a sensor network. Average number of hops, percentage of malicious nodes, Throughput and Time are considered as performance parameters to evaluate the reputation of each node.

\subsection{Genetic Algorithm based Technique}

Qin et al. [36] presented a novel communication protocol for studying the upper bounds on the lifetime of video sensor network. The cost effectiveness makes the network typically small. The proposed algorithm maximizes the network lifetime rather than minimizing the energy depletion of sensors. Some clusters, the number of network lifetime are taken as performance parameters for presenting a graphical simulation which shows N-of-N network lifetime generated by the proposed approach. Some Clusters and Memory used (Bytes), Network lifetime are presented as performance parameters too. It is also found in the paper of Chakraborty et al. [37], genetic algorithm inspired routing algorithm that is termed as GROUP that increases the network performance by mitigating the energy dissipation issues. A linear quantitative structure activity relationship (QSAR) model has been introduced in the paper of uses the concept of genetic algorithm and variable selection tools. Performance analysis of the proposed method obtain the efficient results which shows that GA-MLR model is superior that SW-MLR model. The comparison has been done with respect to standardized residuals and leverage.

\subsection{Neural Networks Based validation Techniques}

Very few techniques have been found for validation of biosensors with Neural Network Concepts. Keller et al. [38] introduced three prototype sensing systems that are used to detect the composition of chemical dyes in liquid and identify the radioactive isotopes respectively. The performance parameters are used to discuss the effectiveness of the proposed system. The study of Singh et al. [39] modeled a photometric biosensor where the performance metrics of the proposed sensor has been evaluated using Artificial Neural Networks. Graphical representation using No of Epochs Vs Mean square error as performance parameters shows 93\% accuracy of the proposed system.

\section{RESEARCH AND DISCUSSION}

The existing studies discussed till the previous section highlights the effectiveness as well as various forms of the latent limitations. There are various forms of the techniques discussed in this manuscript that was found to be used for evaluating the performance of the biological sensors. However, to understand the effectiveness of the existing studies, it is important to review the tradeoff and narrow it down to the research gap. Exploration of the research gap will further enrich the quality of research work in the direction of exploring the best technique of evaluating the performance of biological sensors. The explored research gaps are as follows:

\subsection{Iterative Nature of Algorithm}

The majority of the studies were explored that uses a genetic algorithm, neural network, or game theory for performance evaluation of the sensors. A closer look at all these algorithm types will show that they carry out an iterative form of processing to get the elite outcomes. However the outcomes are not found reliable as adoption of such algorithm are found with certain flaws e.g. i) inability or no assured to find global optimum [31],[32], ii) inability to ensure uniform optimization during response in performance validation of sensors [33], and iii) processing time of algorithm increases with increase in network size [38][39].

\subsection{Less work on Biological Sensor}

Biological sensors (or the wearable sensors) are the very new form of technology that has evolved in last 3-4 years. Hence, a less amount of research work has been witnessed in the literature archival towards biological sensors and majority of the work is carried out for conventional sensors (non-biological-smoke sensor, motion sensor, humidity sensor, etc.). Although there are not much difference in biological and nonbiological in the form of the workability, there is a slight difference between them. Biological sensors (e.g. heart beat sensor or glucose sensor) frequently keeps on capturing the signals and hence have the higher possibility of draining energy more than the conventional sensors. Therefore, the fault tolerances on energy factor of the sensors were never explored in this area. 


\subsection{Less Focus on Computational Model}

Evidence of mathematical or empirical modelling of performance evaluation of biological sensors is missing from the literature. A robust mathematical model will assist to develop the further potential skeleton of the architecture that can furnish better reliability factor in the outcomes. Moreover, although there are various studies towards evaluating the performance of biological sensors, but the works towards validation techniques formulation are quite a few. Less work on reliability analysis on the outcomes are also some of the significant gaps explored in the literatures.

\subsection{Need of More Realistic Performance Parameters}

Very less emphasis is laid towards the realistic assumption of the performance parameters. The existing parameter doesn't seem to consider critical performance about fault tolerance e.g. MAPE (Mean Absolute Percentage Error), fault detection time, alarm type, etc. Hence, until and unless such parameters are not considered for the study, the extent of fault tolerance cannot be studied reliably.

\section{CONCLUSION}

Biological sensors are different from the conventional sensors that are used in habitat monitoring, battlefield etc. Although such types of conventional sensors are also used in a healthcare system, biological sensors are much different from them. Biological sensors are either connected to the superficial part of the body, or it may be even fixed in the internal organs of the body e.g. modern-day pacemakers have sensors. Hence, the data being collected by them should ensure the highest degree of fault tolerance. Therefore, this paper has discussed the significance of the data processing and performance of the biological sensors on various techniques. The prime motive of this paper is to review the existing techniques of performance evaluation for the biological sensor and extract their research gap.

Our future work will be in the direction of resolving the existing problems and will aim to evolve up with a novel framework that can ensure the precise evaluation of the performance of the biological sensors. The secondary aim of the proposed study is to incorporate data fusion technique and auto-associative neural network for evaluating as well as enhancing the performance of biological sensors. The research objectives set for this purpose are as follows e.g. i) to design a technique for enhancing the performance of biological sensors using data fusion technique, ii) to develop a technique that can perform an effective validation of biological sensors using enhanced auto-associative neural network, iii) to further enhance the performance evaluation technique of biological sensors using novel optimization algorithm based on auto-associative neural network, iii) to perform comparative performance analysis of the outcome accomplished from proposed system with the existing one.

\section{REFERENCES}

[1] M. Zourob, "Recognition Receptors in Biosensors," Springer Science \& Business Media, Technology \& Engineering, 2010.

[2] A. Sadana, "Biosensors: Kinetics of Binding and Dissociation Using Fractals: Kinetics of Binding and Dissociation Using Fractals,” Elsevier, 2003.

[3] A. Koyun, et al., "Biosensors and their principles," IntechOpen-A Roadmap of Biomedical Engineers and Milestones, 2012.

[4] B. Lo and G. Z. Yang, "Key technical challenges and current implementations of body sensor networks," Proceeding $2^{\text {nd }}$ International Workshop on Body Sensor Networks, 2005.

[5] L. Schwiebert, et al., "Research challenges in wireless networks of biomedical sensors," ACM-Proceedings of the $7^{\text {th }}$ annual international conference on Mobile computing and networking, pp. 151-165, 2001.

[6] D. W. Carman, et al., "Constraints and approaches for distributed sensor network security (final)," DARPA Project report,(Cryptographic Technologies Group, Trusted Information System, NAI Labs), vol/issue: 1(1), 2000.

[7] R. R. Vis, “Cold chain management using an ultra low power wireless sensor network”, WAMES, 2004.

[8] C. F. García-Hernández, et al., "Wireless sensor networks and applications: a survey," International Journal of Computer Science and Network Security, vol/issue: 7(3), pp. 264-273, 2007.

[9] A. Saeed, et al., “A Scalable Wireless Body Area Network for Bio-Telemetry,” Journal of Information Processing System, vol/issue: 5(2), pp. 77-86, 2009.

[10] S. S. Mohanavalli and S. Anand, "Security architecture for at-home medical care using body sensor network," International Journal of Ad hoc, Sensor \& Ubiquitous Computing, vol. 2, pp. 60-69, 2011.

[11] K. S. Sandeep and Gupta, “A tool for designing high-confidence implantable biosensor networks for medical monitoring," Sigbed Review, vol/issue: 6(2), 2009.

[12] B. Meric, et al., "Indicator-free electrochemical DNA biosensor based on adenine and guanine signals," Electroanalysis-An International Journal Devoted to Fundamental and Practical Aspects Electroanalysis, vol/issue: 14(18), pp. 1245-1250, 2002. 
[13] D. Ivnitski, et al., "Nucleic acid approaches for detection and identification of biological warfare and infectious disease agents,” PubMed, Biotechniques, vol/issue: 35(4), pp. 862-869, 2003.

[14] N. Hamza, et al., "Wireless biomedical system design based on ZigBee technology for autonomous healthcare," Proceedings of International Conference of Communication, Computation, and Power, pp. 15-18, 2009.

[15] C. Lazzari, et al., "Randomized proteomic stratified phase III study of second-line erlotinib (E) versus chemotherapy (CT) in patients with inoperable non-small cell lung cancer (PROSE)," Annual Meeting Proceedings, vol/issue: 31(18), 2013.

[16] P. G. Gopinath, et al., "Microcantilever based Biosensor for Disease Detection Applications," Journal of Medical and Bioengineering, vol. 24, 2015.

[17] J. Homola, “Surface Plasmon Resonance Based Sensors,” Springer Science \& Business Media, 2006.

[18] A. L. A. N. McWhirter and L. Wahlstrom, "The Bene?ts and Scope of Surface Plasmon Resonance-based Biosensors in Food Analysis,” Handbook of Surface Plasmon Resonance, 2008.

[19] S. Jung and M. Yung, "Information Security Applications," Springer Science \& Business Media, 2012.

[20] R. Halir, et al., "Direct and sensitive phase readout for integrated waveguide sensors," IEEE-Photonics Journal, vol/issue: 5(4), 2013.

[21] D. K. C. Wu, et al., "Performance of refractive index sensors based on directional couplers in photonic crystal fibers,” Journal of Lightwave Technology, vol/issue: 31(22), pp. 3500-3510, 2013.

[22] T. Horeman, et al., "Assessment of laparoscopic skills based on force and motion parameters," IEEE Transactions on Biomedical Engineering, vol/issue: 61(3), pp. 805-813, 2014.

[23] B. Mehta and M. E. Zaghloul, "Tuning the scattering response of the optical nano antennas using grapheme," IEEE Photonics Journal, vol/issue: 6(1), pp. 1-8, 2014.

[24] E. Blasch and J. Gainey, "Feature Based Biological Sensor Fusion,” International Conference on Information Fusion, pp. 702-709, 1998.

[25] M. N. Rahman and M. A. Matin, "Efficient algorithm for prolonging network lifetime of wireless sensor networks," IEEE-Tsinghua Science \& Technology, vol/issue: 16(6), pp.561-568, 2011.

[26] W. Hu, et al., "A virtual sensor scheduling framework for heterogeneous wireless sensor networks," IEEE 38th Conference on Local Computer Networks, pp. 655-658, 2013.

[27] J. Yuan, et al., "Gold elliptic nanocavity arrays biosensor with high refractive index sensitivity based on twophoton nanolithography,” IEEE-Photonics Journal, vol/issue: 7(1), 2014.

[28] C. A. Chen, et al., "Ubiquitous Monitoring for Industrial Cyber-Physical Systemsover Relay Assisted Wireless Sensor Networks,” IEEE-Transactions on Emerging Topics in Computing, 2015.

[29] I. Nevat, et al., "Estimation of Spatially Correlated Random Fields in Heterogeneous Wireless Sensor Networks," IEEE Transactions on Signal Processing, vol/issue: 63(10), pp. 2597-2609, 2015.

[30] V. Kotari and K. C. Chang, "Epidemic Biological Data Fusion with Ad-hoc Sensor Networks," Communications in Information Science and Management Engineering, 2012.

[31] V. Rangelova and D. Tsankova, "Fuzzy modeling the influence of temperature on tissue biosensor for measurement of dopamine,” IEEE Conference on Control \& Automation, pp. 1-5, 2007.

[32] P. K Singh, et al., "Optimized and Self-Organized Fuzzy Logic Controller for $\mathrm{pH}$ Neutralization Process," International Journal of Intelligent Systems \& Applications, vol/issue: 5(12), 2013.

[33] T. Haider and M. Yusuf, "A fuzzy approach to energy optimized routing for wireless sensor networks," International Arab Journal of Information technology, vol/issue: 6(2), pp. 179-185, 2009.

[34] G. Koltsidas and F. N. Pavlidou, "A game theoretical approach to clustering of ad-hoc and sensor networks," Telecommunication Systems vol/issue: 47(1-2), pp. 81-93, 2011.

[35] A. Agah and S. K. Das, "Preventing DoS Attacks in Wireless Sensor Networks: A Repeated Game Theory Approach,” International Journal of Network Security, vol/issue: 5(2), pp.145-153, 2007.

[36] M. Qin and R. Zimmermann, "Maximizing video sensor network lifetime through genetic clustering," USC Technical Report USC-CS-TR05-844, University of Southern California, 2005.

[37] A. Chakraborty, et al., "A Genetic algorithm inspired routing protocol for wireless sensor networks," International Journal of Computational Intelligence Theory and Practice, vol/issue: 6(1), pp.1-8, 2011.

[38] P. E. Keller, et al., “Three neural network based, sensor systems for environmental monitoring,” IEEE Electro/94 International. Conference Proceedings. Combined, pp. 377-382, 1994.

[39] P. Singh and H. M. Rai, "Performance analysis of photometric strain biosensor for bones using artificial neural network,” International Journal of Computer Applications, vol/issue: 54(8), pp. 16-19, 2012. 


\section{BIOGRAPHIES OF AUTHORS}

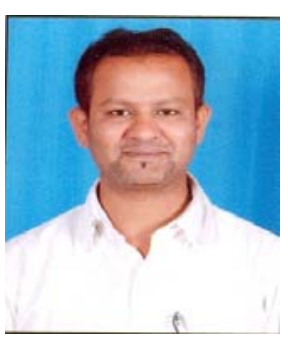

Subhas A. Meti received the B.E. degree in Electronics and Communication Engineering from the Karnataka university Dharwad, Karnataka in 2000, the M.Tech degree in Instrumentation Engineering form Swami Ramanand Teerth Marathwada University of Nanded, Maharashtra in 2007. Presently pursuing the Ph.D. degree in Electrical Engineering from the Visvesvaraya Technological University Belgaum, Karnataka. Currently, He is working as an assistant Professor of Instrumentation Engineering at B V B college of Engineering and Technology, Hubli. His teaching and research areas include instrumentation, Multisensor data fusion, Soft computing.

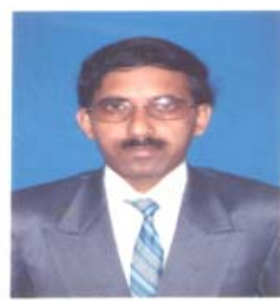

V G Sangam received the B.E. degree in Instrumentation Engineering from the Mysore university, Karnataka in 1989, the M.E degree in Instrumentation Engineering form Mysore university, Karnataka in 2000, and the Ph.D. degree in Instrumentation Engineering from SwamiRamanand Teerth Marathwada University of Nanded, Maharashtra in 2007, respectively.. Currently, He is working as Professor of Electrical science at Adama science \& Technology University, Ethiopia. His teaching and research areas include instrumentation, Biosensor, process instrumentation and Control system. 